OPEN ACCESS

Edited by:

Helge Frieling,

Hannover Medical School, Germany

Reviewed by:

Michael Kluge,

University of Leipzig, Germany

Erik Gunnar Jönsson,

University of Oslo, Norway

*Correspondence:

Chen Zhang

zhangchen645@gmail.com

${ }^{\dagger}$ These authors have contributed equally to this work and share first authorship

Specialty section:

This article was submitted to

Molecular Psychiatry,

a section of the journal

Frontiers in Psychiatry

Received: 10 September 2020

Accepted: 27 October 2020

Published: 25 November 2020

Citation:

Fang $X, Y u L$, Wang D, Chen Y,

Wang $Y$, Wu Z, Liu R, Ren J, Tang $W$

and Zhang C (2020) Association

Between SIRT1, Cytokines, and Metabolic Syndrome in Schizophrenia Patients With Olanzapine or Clozapine

Monotherapy.

Front. Psychiatry 11:602121.

doi: 10.3389/fpsyt.2020.602121

\section{Association Between SIRT1, Cytokines, and Metabolic Syndrome in Schizophrenia Patients With Olanzapine or Clozapine Monotherapy}

\author{
Xinyu Fang ${ }^{1,2 \dagger}$, Lingfang $\mathrm{Yu}^{1+}$, Dandan Wang ${ }^{1 \dagger}$, Yan Chen ${ }^{1}$, Yewei Wang ${ }^{1}$, Zenan $\mathrm{Wu}^{1}$, \\ Ruimei Liu ${ }^{1}$, Juanjuan Ren ${ }^{1}$, Wei Tang ${ }^{3}$ and Chen Zhang ${ }^{1 *}$

\begin{abstract}
${ }^{1}$ Schizophrenia Program, Shanghai Mental Health Center, Shanghai Jiao Tong University School of Medicine, Shanghai, China, ${ }^{2}$ Affiliated Nanjing Brain Hospital, Nanjing Medical University, Nanjing, China, ${ }^{3}$ The Affiliated Kangning Hospital of Wenzhou Medical University, Wenzhou, China
\end{abstract}

Objective: Previous studies consistently showed the interaction between Sirtuin 1 (SIRT1) and immune inflammation is significantly related to metabolic abnormalities, but their role in the pathogenesis of metabolic syndrome caused by second-generation antipsychotics (SGAs) in schizophrenia patients largely remains unknown. Hence, the present study aimed to fill this gap.

Methods: A total of 54 schizophrenia patients with olanzapine or clozapine monotherapy [metabolic syndrome (MetS)/non-MetS patients, 27/27] and 67 healthy subjects were recruited in the present study. The Positive and Negative Syndrome Scale was used, and the plasma levels of SIRT1, interleukin 6 (IL-6), IL-8, IL-10, and tumor necrosis factor $\alpha$ (TNF- $\alpha$ ) were measured.

Results: The results showed that schizophrenia patients treated with olanzapine or clozapine (both MetS and non-MetS groups) had significantly higher plasma levels of IL-6, IL-10, and TNF- $\alpha$ compared to normal controls (all $P<0.05$ ). Moreover, the MetS patients exhibited markedly lower plasma levels of SIRT1 and higher plasma levels of IL-6 than non-MetS patients and normal controls (all $P<0.05$ ). However, there were no significant differences in IL-8 levels between groups. Our correlation analysis showed that SIRT1 was significantly correlated with diastolic blood pressure, triglyceride, and high-density lipoprotein cholesterol in schizophrenia patients. The stepwise logistic regression analysis further identified the IL-6 $\times$ SIRT1 $(\beta=-0.463, t=10.040, P=0.002)$ as the influencing factor for the MetS in the patients.

Conclusion: Our preliminary findings suggest that SIRT1 interacted with inflammatory cytokines associated with MetS in schizophrenia patients treated with SGA monotherapy.

Keywords: schizophrenia, SIRT1, cytokine, metabolic syndrome, olanzapine, clozapine 


\section{INTRODUCTION}

Schizophrenia is a severe and debilitating psychiatric disorder characterized by a wide range of symptoms, including positive symptoms, negative symptoms, and impaired cognition (1). Antipsychotic drugs have been widely used to treat schizophrenia patients since the advent of chlorpromazine uniformly has alleviated positive symptoms in the 1950s (2). In recent decades, second-generation antipsychotics (SGAs) have been more frequently prescribed for schizophrenia patients profiting from the lower risk of extrapyramidal symptoms and tardive dyskinesia compared to the first-generation antipsychotics (FGAs) (3). Unfortunately, most SGAs, led by clozapine and olanzapine, cause serious metabolic adverse effects including weight gain, abdominal obesity, hyperglycemia, hypertension, and dyslipidemia (4). The Clinical Antipsychotic Trials of Intervention Effectiveness trial reported the prevalence of metabolic syndrome (MetS) to be around $40 \%$ in schizophrenia patients based on the National Cholesterol Education ProgramAdult Treatment Panel III criteria (NCEP-ATP III) (5). Our recent study supports that schizophrenia patients receiving olanzapine monotherapy more than 2 years had a $44 \%$ risk of developing MetS (2). MetS occurring in schizophrenia patients usually leads to several negative outcomes such as poor cognitive function, non-compliance, higher residual psychopathology (6), lower life expectancy, and increased mortality rates (7). Therefore, searching for molecular markers of MetS induced by SGAs is of great significance to elucidate its pathological mechanism.

In the past decade, emerging research has explored the molecular mechanisms underlying the possible processes for SGA-induced metabolic abnormalities. As SGAs act at multiple neurotransmitter receptors, including dopamine $\mathrm{D}_{1}, \mathrm{D}_{2}, \mathrm{D}_{3}$, and $\mathrm{D}_{4}$; adrenergic $\alpha 1$ and $\alpha 2$; serotonin $5 \mathrm{HT}_{2 \mathrm{~A}}$ and $5 \mathrm{HT}_{2 \mathrm{C}}$; and muscarinic and histamine $\mathrm{H}$ receptors compared to FGAs, the researchers found that these different receptors may be associated with metabolic abnormalities associated with SGAs (8), especially $5 \mathrm{HT}_{2 \mathrm{~A}}, 5 \mathrm{HT}_{2 \mathrm{C}}$, and $\mathrm{H}$ receptors, which has an effect on food intake via the hypothalamus (9). In addition, pathways related to altered ghrelin and leptin release, dysfunction of mitochondria, oxidative stress reactions, dysfunctions in the autonomic nervous system activity, altered gut microbiome, and aberrant immuneinflammatory system have also been implicated as important contributing processes leading to MetS with the use of SGAs $(2,10)$. However, relevant debate and exploration are ongoing, and new molecular markers responsible for the SGAs-caused MetS need to be developed.

Sirtuin 1 (SIRT1) is a highly conserved $\mathrm{NAD}^{+}$-dependent protein deacetylase and is widely distributed in the body. Emerging evidence has shown that SIRT1 has important effects on the regulation of lipid and glucose metabolism $(11,12)$. Early studies indicated that SIRT1 gene expression in peripheral blood and adipose tissue was significantly diminished in obese subjects compared to control subjects (13), and patients with fatty liver or diabetes also showed reduced plasma levels of SIRT1 $(14,15)$. In animal models, it was demonstrated that mice with SIRT1 adipocyte-specific knockout gained weight rapidly, along with glucose intolerance and insulin resistance compared with control mice (16), while the SIRT1 agonist resveratrol could prevent or ameliorate glucose and lipid metabolic disturbance (17). Furthermore, a recent study found oral resveratrol supplementation could improve MetS features in obese patients (18). Taken together, down-regulation of the SIRT1 may contribute to obesity-associated metabolic abnormalities. However, little is known about the relationship between SIRT1 and SGAs-caused MetS in schizophrenia patients. Interestingly, recent research showed that paliperidone, one of the SGAs, could significantly decrease the expression and production of SIRT1 in vitro (19). Thus, the role of SIRT1 on MetS induced by SGAs and its potential mechanism require further investigation.

Chronic and low-grade inflammation is invariably a common feature of the MetS and obesity, where inflammatory signals originate from within visceral adipose tissue in addition to the peripheral system $(20,21)$. SGAs also potentiate aberrant peripheral levels of inflammatory markers $(22,23)$, which are associated with their effect on weight gain (22). A study of RNA sequencing revealed that pathways related to the immune inflammatory system were highly enriched with differential expression genes in antipsychotics-caused obesity in patients with schizophrenia (24). Furthermore, our recent work indicated that schizophrenia patients with MetS present an increased expression and production of inflammatory indices $(2,25)$. This phenomenon has also been supported by other studies showing that altered cytokines levels were significantly associated with the components of MetS induced by SGAs (26-28). In addition, previous clinical observations demonstrated that supplement anti-inflammatory therapy can correct SGAs-caused metabolic abnormalities in schizophrenia patients and mice $(29,30)$.

Interestingly, previous studies have shown that SIRT1 played a vital role in influencing immune responses (31), and SIRT1 can regulate immune responses directly through deacetylation of some key transcriptional factors or indirectly through metabolic pathways (32). A recent study found that diabetic patients and obese subjects had higher serum IL-6 but lower SIRT1 levels compared to healthy subjects (33). Further evidence from another recent study also showed SIRT1 agonist resveratrol could significantly inhibit the immune inflammation of the body and improve the dysfunction of glycolipid metabolism $(32,34)$. Thus, altered SIRT1 that interacted with immune inflammation may be associated with MetS caused by SGAs. However, no study to date has explored this issue.

Clozapine and olanzapine are more notorious for causing MetS compared to other SGAs. In light of the abovementioned findings, the present study aimed to investigate the association between SIRT1, inflammatory cytokine levels, and MetS in schizophrenia patients with a long-term olanzapine or clozapine monotherapy and to evaluate if any of such association was mediated independently or interactively in relation to MetS in this population. We hypothesized that the reduced SIRT1 plasma levels may be associated with altered inflammatory cytokines in schizophrenia, and the interaction between SIRT1 and cytokines may contribute to the MetS caused by SGAs in schizophrenia patients. 


\section{METHODS}

\section{Participants}

A total of 54 schizophrenia patients (27 patients with MetS and 27 patients with non-MetS) with age range from 18 to 50 years were included in the present study. All schizophrenia patients were recruited from Shanghai Mental Health Center and met the following criteria: (1) had been diagnosed with schizophrenia according to the Diagnostic and Statistical Manual of Mental Disorders, Fourth Edition (DSM-IV) (corresponding to the International Classification of Diseases, 10th Revision item F20), diagnoses were made using modified sections of the Structured Clinical Interview for $D S M-I V$ disorders by at least 2 experienced psychiatrists; (2) age 18-50 years, Han Chinese; (3) had a junior high school education or above; and (4) patients were receiving clozapine or olanzapine monotherapy for at least 6 months. The exclusion criteria included the following (1) had head trauma with residual effects, neurological disorders; (2) pregnant or breastfeeding; (3) had other diagnosed psychiatric disorder besides schizophrenia or a lifetime substance abuse/dependence disorder; (4) had a recent history of highgrade fever/infection within the past 1 month or any comorbid medical disease that could potentially influence the immune system; and (5) the patients received no drug(s), including statins or anti-diabetes medication, with well-known effects on the study parameters. We also recruited 67 healthy control subjects with age range from 19 to 50 years, who were screened by a specialized psychiatrist using the Structured Clinical Interview for DSM$I V-T R$ Axis I Disorders-Patient Edition. All control subjects were self-reported to have no hypertension, hyperglycemia, and hyperlipidemia-related metabolic diseases.

This study was performed in strict accordance with the Declaration of Helsinki and other relevant national and international regulations. All procedures for this study were reviewed and approved by the institutional review boards of the Shanghai Mental Health Center, and written informed consent was obtained from each participant before the commencement of this study.

\section{Clinical Measurement}

All participants were interviewed face-to-face. Basic sociodemographic data such as age, sex, education level, weight, height, body mass index (BMI), and clinical data (age of onset, disease course, drug type) were recorded by trained psychiatrists. The 30-item Positive and Negative Syndrome Scale (PANSS) was assessed by two trained psychiatrists, who had worked at least 5 years in clinical practice. Repeated assessment for the PANSS total score maintained an interrater correlation coefficient $>0.8$. The three-factor PANSS model was used to assess the psychopathology of schizophrenia patients, consisting of positive symptoms $(\mathrm{P})$, negative symptoms $(\mathrm{N})$, and general psychiatric symptoms $(\mathrm{G})$.

\section{Anthropometric and Metabolic Parameters}

Based on the definition by the NCEP-ATP III, the MetS was diagnosed by any three of the following criteria: (1) a waist circumference $\geq 90 \mathrm{~cm}$ in Chinese men and $\geq 80 \mathrm{~cm}$ in
Chinese women; (2) triglyceride (TG) $\geq 1.7 \mathrm{mmol} / \mathrm{L}$; (3) highdensity lipoprotein cholesterol (HDL) $<1.0 \mathrm{mmol} / \mathrm{L}$ in men and $<1.3 \mathrm{mmol} / \mathrm{L}$ in women; (4) blood pressure $\geq 130 / 85$ $\mathrm{mmHg}$; or (5) fasting glucose (GLU) $\geq 5.6 \mathrm{mmol} / \mathrm{L}$, which is considered as the best MetS criterion for a Chinese population (2).

Blood samples from the patients were collected between 6:00 and 9:00 A.M. following an overnight fast. The serum was separated, aliquoted, and stored at $-80^{\circ} \mathrm{C}$ until used for the assay. Serum fasting GLU, TG, and HDL levels were measured using an automatic Biochemical Analyzer (HITACHI 7170A, Hitachi, Ltd., Tokyo, Japan). Blood pressure including systolic blood pressure and diastolic blood pressure (DBP) was measured using an automated blood pressure machine before the blood collection. The waist circumference in patients was measured between the lower rib margin and the iliac crest, after a normal expiratory breath as described in our previous article $(35,36)$.

\section{Plasma SIRT1 and Cytokines Measurement}

A total of $10 \mathrm{~mL}$ fasting blood samples were collected from 54 patients and 67 healthy controls between 6:00 and 9:00 A.M. The plasma was separated by centrifugation at 3,000 $\mathrm{r} / \mathrm{min}$ for $15 \mathrm{~min}$ at $4^{\circ} \mathrm{C}$ and stored at $-80^{\circ} \mathrm{C}$ until used for assay. Plasma levels of SIRT1, interleukin 6 (IL-6), IL8 , IL-10, and tumor necrosis factor $\alpha$ (TNF- $\alpha$ ) were using enzyme-linked immunosorbent assay kits (RayBiotech, Norcross, GA). The limitation of detection was $7.8 \mathrm{pg} / \mathrm{mL}$ for SIRT1, $1.0 \mathrm{pg} / \mathrm{mL}$ for SIRT1, and $0.5 \mathrm{pg} / \mathrm{mL}$ for IL-6, IL-8, and IL10. The experiment performers were blind to all the clinical data. The reproducibility of the assay was tested prior to the measurement of the samples, and the interassay coefficient of variation was $<5 \%$.

\section{Data Analysis}

The Statistical Package for the Social Sciences version 23.0 was used for data analysis. First, the Student $t$-test or the analysis of variance or the $\chi^{2}$ tests were used to compare the differences between groups as appropriate. The analysis of covariance was further performed to control potential confounding factors, such as age, sex, and education levels. The Fisher least significant difference (LSD) was used for multiple comparisons. Second, we correlated the plasma levels of SIRT1 and cytokines in whole schizophrenia patients. Third, Pearson correlation analysis was used to test the correlation between MetS components and SIRT1 or cytokines, and partial correlation analysis was further conducted to control confounding factors. Finally, stepwise logistic regression analysis was performed to explore the links between plasma levels of SIRT1, cytokines, and MetS and their interaction effects in the patients. MetS were selected as dependent variables, whereas altered plasma values (cytokines and SIRT1) between groups, as well as significant interactions between cytokines and SIRT1, were assessed as independent variables. All statistical tests were two-tailed, and statistical significance was set at $\alpha \leq 0.05$. 


\section{RESULTS}

\section{Demographic and Clinical Characteristics Among Control Subjects and MetS and Non-MetS Patients With Schizophrenia}

Table 1 shows the demographic data of healthy controls and MetS and non-MetS patients with schizophrenia. There were no significant differences in age, sex, and height among those three groups (all $P>0.05$ ), but there were differences in education levels, weight, and BMI $(F=4.772, P=0.011 ; F=9.368, P<$ $0.001, F=13.468, P<0.001$, respectively). After further LSD correction for multiple comparisons, we found there were no significant differences in BMI and weight between non-MetS patients and normal controls (both $P>0.005$ ). Both MetS and non-MetS patients had lower education levels compared to normal controls $(P=0.004, P=0.027$, respectively), but no difference between MetS and non-MetS patients $(P>0.05)$. For clinical characteristics between Mets and non-MetS patients, the results showed no significant differences in age at onset, family history of mental illness, and duration of monotherapy with olanzapine or clozapine and olanzapine equivalents calculated based on defined daily doses (37) between those two patient groups (all $P>0.05$ ). Patients with MetS had a longer disease courses $(t=2.662, P=0.010)$ compared to non-MetS patients. Our results also showed significant differences in PANSS total scores and PANSS-G subscale scores (PANSS total score: $t$ $=2.248, P=0.029$; PANSS-G: $t=2.275, P=0.027)$, but no differences in PANSS-P, PANSS-N subscale scores between MetS and non-MetS patients (both $P>0.05$ ). In addition, schizophrenia patients with MetS had higher waist circumference $(t=3.071, P=0.003)$, DBP $(t=2.955, P=0.005)$, fasting GLU $(t=2.108 ; P=0.040)$, and TG $(t=5.827 ; P<0.001)$, but lower serum HDL levels $(t=2.306 ; P=0.025)$ compared to non-MetS patients.

\section{Comparisons of Cytokines and SIRT1 Among MetS Patients, Non-MetS Patients, and Control Subjects}

As shown in Table 1, plasma levels of IL-6, IL-10, TNF- $\alpha$, and SIRT1 varied significantly across MetS patients, non-MetS patients, and control subjects $(F=37.205, P<0.001 ; F=$ 24.114, $P<0.001 ; F=39.744, P<0.001 ; F=11.058, P<$ 0.001 , respectively). When age, sex, and education levels were added as covariates, the differences in IL- 6 , TNF- $\alpha$, and SIRT1 among those three groups remained significant $(F=4.091, P=$ $0.025 ; F=5.530, P=0.008 ; F=6.973, P=0.003$, respectively), but the difference in IL-10 did not $(P>0.05)$. There was no significant difference in plasma levels of IL-8 among those three groups $(P<0.05)$. After the post hoc comparisons using LSD were performed, our results demonstrated that both MetS and non-MetS patients had notably higher IL-6 levels compared to normal controls (both $P<0.001$ ), and increased levels of IL-6 were also more pronounced in MetS patients compared to nonMetS patients $(P=0.032)$. In addition, the results indicated that both MetS and non-MetS patients had significantly higher IL-10 and TNF- $\alpha$ compared to control group (both $P<0.001$ ), but no significant differences were found between the two patient groups $(P>0.05)$. Interestingly, plasma levels of SIRT1 were markedly lower in MetS patients compared to non-MetS patients $(P=$ $0.001)$ and control $(P<0.001)$, whereas there was no significant difference between non-MetS patients and controls $(P>0.05$; Figure 1).

\section{Correlations of SIRT1 and Cytokines in Schizophrenia Patients}

In all patients with schizophrenia, our results showed that plasma levels of SIRT1 were negatively correlated with IL-6 $(r=-0.345$, $P=0.011), \mathrm{IL}-10(r=-0.276, P=0.043)$, and TNF- $\alpha(r$ $=-0.393, P=0.003)$. The correlations between SIRT1 and IL- 6 or TNF- $\alpha$ remained significant when partial correlation was performed to control for confounding factors including age, sex, and BMI $(r=-0.329, P=0.018 ; r=-0.396 ; P=$ 0.004 , respectively), but SIRT1 and IL-10 did not. No significant correlation between plasma levels of SIRT1 and IL-8 were found among all patients $(r=-0.128, P=0.358)$.

\section{Risk Factors for MetS and Its Components in Schizophrenia Patients}

To identify which MetS components were most strongly associated with SIRT1 or cytokines in schizophrenia patients, the Pearson correlation analysis was performed. The results showed that DBP $(r=-0.406, P=0.002)$ and TG $(r=-0.346, P=$ 0.010 ) were inversely related with SIRT1 levels in the patients, whereas HDL was positively correlated with SIRT1 levels in the patients $(r=0.292, P=0.032)$. These correlations still remained significant after controlling for age, sex, and BMI $(r$ $=-0.386, P=0.005 ; r=-0.351, P=0.012 ; r=0.276, P=$ 0.050 , respectively) (Figure 2). Interestingly, our results indicated that IL-6 and IL-10 levels were both correlated with BMI in schizophrenia patients $(r=0.233, P=0.010 ; r=0.204, P=$ 0.025 , respectively). However, there were no other significant correlations between SIRT1, IL-6, and IL-10 levels and MetS components in schizophrenia patients (all $P>0.05$ ). To further explore the interaction of SIRT1 and cytokines on MetS in schizophrenia patients, the stepwise multiple regression analysis was conducted, with $P$-value criteria of 0.01 and 0.05 for entry and removal, respectively. Our results identified the IL- $6 \times$ SIRT1 ( $\beta=-0.463, t=10.040, P=0.002)$ as the influencing factor for the MetS in schizophrenia patients.

\section{DISCUSSION}

To the best of our knowledge, this is the first study that aimed to investigate the association of SIRT1 and MetS caused by SGAs in schizophrenia patients and further to identify the potential interaction of SIRT1 and inflammatory cytokines on the MetS. The main findings in the present study are as follows: (1) schizophrenia patients treated with olanzapine or clozapine (both MetS and non-MetS groups) had significantly higher plasma levels of IL-6, IL-10, and TNF- $\alpha$ compared to normal controls. (2) Moreover, our preliminary study showed that patients exhibited markedly lower plasma levels of SIRT1 and higher plasma levels 
TABLE 1 | Demographic and clinical characteristics among control subjects, MetS and non-MetS patients with schizophrenia.

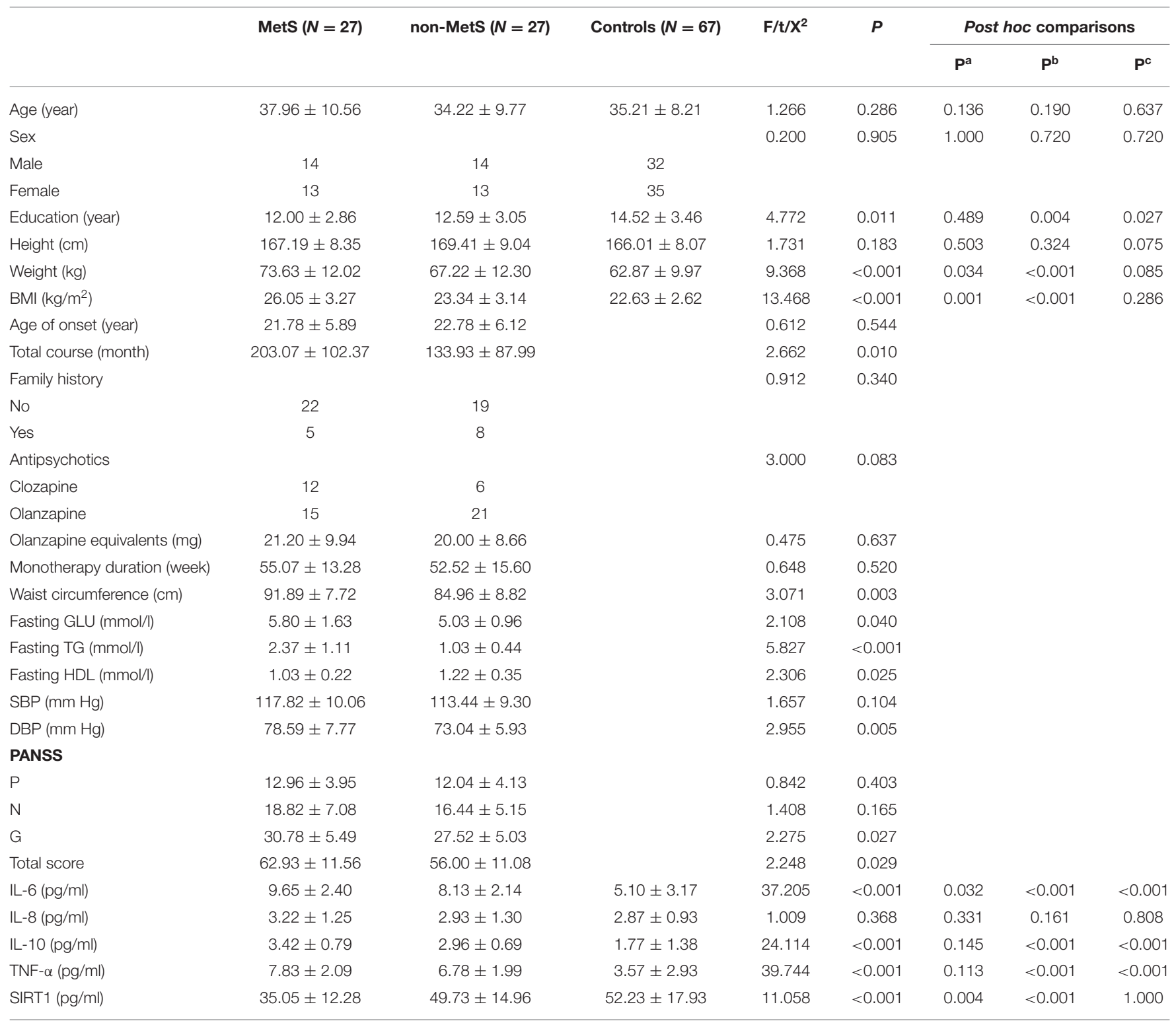

Data presented as Mean \pm SD or N; Olanzapine equivalents were calculated based on defined daily doses (DDDs); BMI, Body Mass Index; IL, Interleukin; TNF, Tumor Necrosis Factor; SIRT1, Sirtuin 1, GLU, glucose; TG, Triglyceride; HDL, High density lipoprotein; SBP, Systolic Blood Pressure; DBP, Diastolic Blood Pressure; PANSS, Positive and Negative Syndrome Scale; P, positive symptoms; $N$, negative symptoms; G, general psychiatric symptoms.

$P^{a}$ : MetS vs. non-MetS; $P^{b}$ : MetS vs. controls; $P^{c}$ : non-MetS vs. controls.

of IL-6 than non-MetS patients and normal controls. (3) The stepwise logistic regression analysis identified the IL- $6 \times$ SIRT1 as the influencing factor for the MetS in schizophrenia patients.

The high risk for the occurrence of MetS in schizophrenia patients may be caused by the combination of multiple factors; coshared genetics and possible risk gene pathway partially explain those comorbidities (38). The SIRT1 gene is widely recognized as an important role in regulating metabolic status. Ample evidence supports the adipose-specific knockdown of SIRT1 results in obesity, dyslipidemia, and insulin resistance $(39,40)$. Moreover, SIRT1 gene was also reported significantly associated with ambulatory blood pressure level in patients with hypertension (41). Although two early studies explore the relationship between SIRT1 gene and susceptibility to schizophrenia, and both suggested that altered expression, mutations, and (or) polymorphisms of SIRT1 might be involved in the pathogenesis of schizophrenia (42), no study to date has explored the relationship between SIRT1 gene and metabolic dysregulation in schizophrenia patients. Interestingly, increased peripheral levels of SIRT1 were frequently reported to reduce the accumulation of fat and decrease the risk of obesity, hypertension, hyperlipidemia, and diabetes; the low expression of SIRT1 therefore predisposes to the development of MetS (43). Thus, we hypothesized that the high incidence of metabolic 

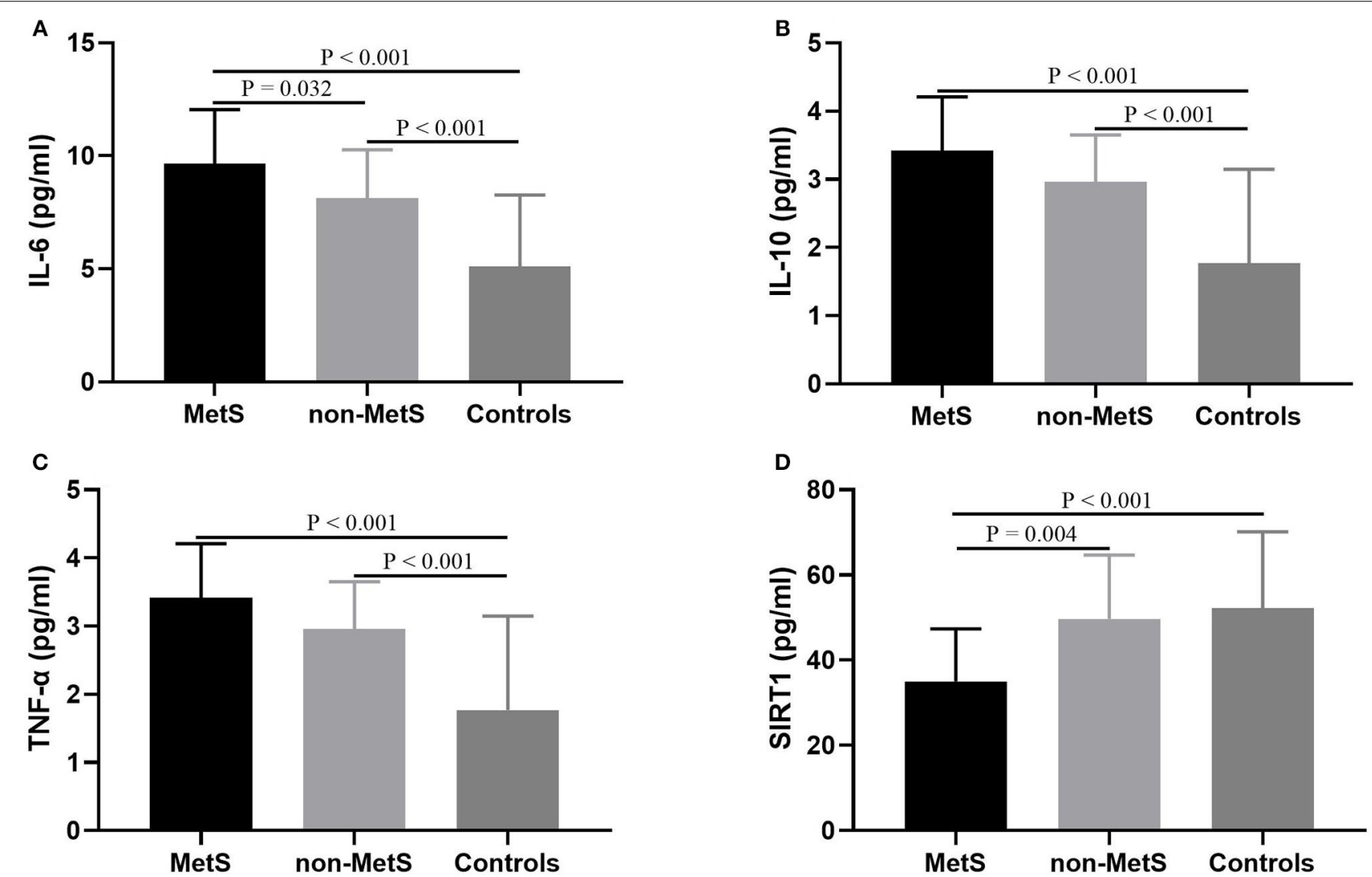

FIGURE 1 | Comparisons of SIRT1 and cytokines levels among MetS patients, non-MetS patients, and control subjects. Each bar represents the mean level of SIRT1 or cytokines. Error bars represent the standard deviation (SD). P-value was calculated by adjusting for the characteristics including age, sex, and body mass index.

(A) Comparison of IL-6 levels among three groups; (B) Comparison of IL-10 levels among three groups; (C) Comparison of TNF- $\alpha$ levels among three groups;

(D) Comparison of SIRT1 levels among three groups. MetS, metabolic syndrome; SIRT1, sirtuins; IL, interleukin; TNF, tumor necrosis factor.

A

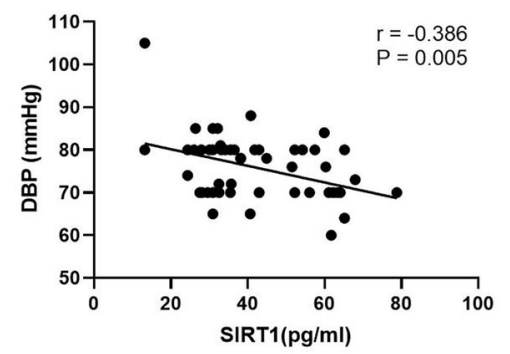

B

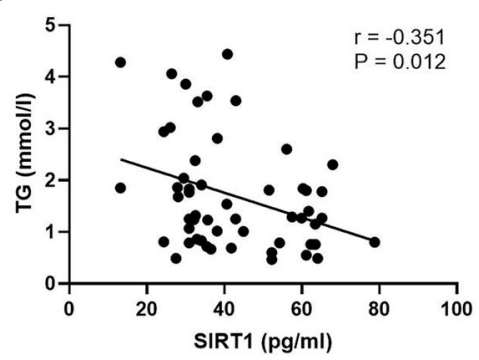

C

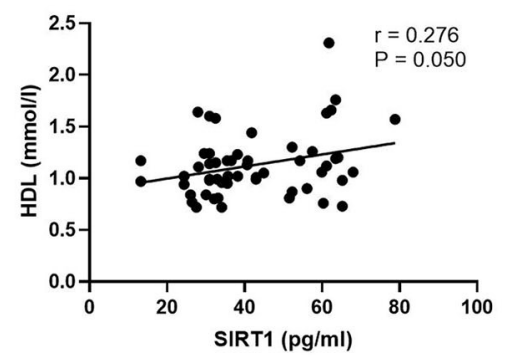

FIGURE 2 | Partial correlation analysis of SIRT1 and DBP, TG, and HDL in schizophrenia patients. P-value was calculated by adjusting for the characteristics including age, sex, and body mass index. (A) Correlation between SIRT1 and DBP in schizophrenia patients; (B) Correlation between SIRT1 and TG in schizophrenia patients; (C) Correlation between SIRT1 and HDL in schizophrenia patients. SIRT1, sirtuins; DBP, diastolic blood pressure; TG, triglyceride; HDL, high-density lipoprotein cholesterol.

dysregulation in schizophrenia patients may be related to the abnormality of SIRT1.

In the present study, we found that MetS patients with SGA monotherapy had markedly lower plasma SIRT1 levels compared to non-MetS patients and healthy controls. Although no study to date has explored the association of peripheral SIRT1 and metabolic abnormalities in schizophrenia, an early study demonstrated that atypical antipsychotics could downregulate the expression of SIRT1 and interfere with the SIRT1 pathway (19). Combined with previous evidence, the findings of the present study suggest that the MetS caused by atypical antipsychotics may be related to its regulation of SIRT1. To further figure out which metabolic components were significantly associated with SIRT1 in schizophrenia patients, we performed 
correlation analysis and found that SIRT1 was inversely or positively associated with DBP, TG, and HDL, but not GLU. Early study conducted in patients with type 2 diabetes also demonstrated the association of SIRT1 gene and DBP, but not other type 2 diabetes-related traits $(41,44)$. In addition, previous study shows that patients with arterial stiffness and hypertension had reduced serum levels of SIRT1 compared to normal subjects (44). Qing Gan Zi Shen Tang, as a famous traditional Chinese medicine for its efficacy in treating hypertension, obesity, and hyperlipidemia, and that therapeutic role was recently demonstrated through up-regulation of SIRT1 (19). Hence, pharmacological activation of SIRT1 may be an effective therapeutic strategy for hypertension and hyperlipidemia, and the SIRT1 activator resveratrol further confirmed this hypothesis (45). Although our preliminary study shows no significant correlation between plasma levels of SIRT1 and fasting glucose in schizophrenia patients treated with SGAs, many existing studies to date revealed that reduced SIRT1 is involved in the pathogenesis of hyperglycemia and diabetes (46-49). The nonsignificant correlation in the present study may be due to our insufficient sample size, and further research with larger sample size is warranted to clarify this.

A promising mechanism underlying the SIRT1 involved in MetS in schizophrenia patients may be associated with the activation of the immune inflammatory system. Substantial evidence supports the activation of inflammatory pathways could disrupt normal metabolism and contribute to the development of metabolic dysfunction $(22,50,51)$. In the present study, we found that schizophrenia patients with olanzapine or clozapine monotherapy exhibited increased plasma levels of IL-6, IL-10, and TNF- $\alpha$, which was also observed in many previous studies $(25,52)$. Moreover, increased plasma levels of IL-6 and IL-10 were more remarkable in patients with MetS compared to nonMetS patients. Our findings are consistent with some previous studies demonstrating that schizophrenia patients with obesity and MetS displayed markedly elevated levels of proinflammatory factors $(2,29,53,54)$. Interestingly, numerous studies have indicated that SIRT1 plays an important role in immune regulation, and the interaction between SIRT1 and immune inflammation is significantly related to metabolic abnormalities $(55,56)$. We extended this association to show that the SIRT1 interacting with IL-6 as the influencing factor for the MetS in schizophrenia patients. It is believed that $\omega-3$ polyunsaturated fatty acids (PUFAs) exhibit significant effects on improved insulin sensitivity and obesity, and early study further revealed the effect of $\omega$-3 PUFAs on metabolic is mainly exerted by activating SIRT1 and ensures inhibition of the release of proinflammatory cytokine including IL- 6 and TNF- $\alpha(57,58)$. However, the SIRT1 inhibitor could abrogate the suppression of TNF- $\alpha$ and IL- 6 production by $\omega$-3 PUFAs (57). Taken together, decreased SIRT1 may bring out MetS in schizophrenia patients through the activation of inflammatory pathways.

Our preliminary findings must be interpreted with some limitations. The main limitations are obviously the crosssectional nature of our study, which preclude us to make firm conclusions about the directionality of SIRT1, inflammatory cytokine on MetS caused by SGAs in schizophrenia patients.
In the present study, only healthy subjects without MetS were included as controls, whereas MetS subjects without mental illness and not using antipsychotics were not recruited in the present study. The lack of such a control group makes it difficult to distinguish between drug-induced mechanisms for MetS and processes that operate independently of drugs. Furthermore, because of the lack of drug-use data for all the patients before they were treated with olanzapine or clozapine, we could not assess previous treatment and its interference with the present results, which was another limitation of our study. In addition, although olanzapine and clozapine are the two most obvious atypical antipsychotics that cause MetS, the small sample size does not allow our study to be further stratified by drugs. Therefore, a longitudinal study with larger study samples and with best measure to reduce the confounding effect is required to evaluate causal relations between SIRT1, immune inflammation, and MetS in schizophrenia patients.

Taken together, we performed a clinical study to examine the association between SIRT1, inflammatory cytokines, and MetS in schizophrenia patients receiving long-term olanzapine or clozapine monotherapy. Our preliminary study provides evidence suggesting that schizophrenia patients with MetS exhibited reduced plasma SIRT1 but increased inflammatory cytokines levels compared to the non-MetS patients. Moreover, the SIRT1 interacting with IL-6 was significantly responsible for the occurrence of the MetS caused by SGAs in schizophrenia patients. Future longitudinal studies are needed to confirm the role of SIRT1 involved in the pathogenesis of MetS in schizophrenia patients and to further reveal its immuneinflammatory mechanism.

\section{DATA AVAILABILITY STATEMENT}

The raw data supporting the conclusions of this article will be made available by the authors, without undue reservation.

\section{ETHICS STATEMENT}

The studies involving human participants were reviewed and approved by Institutional Review Boards of the Shanghai Mental Health Center. The patients/participants provided their written informed consent to participate in this study.

\section{AUTHOR CONTRIBUTIONS}

XF and CZ: conceptualization and funding acquisition. XF, LY, and DW: methodology, formal analysis, and writing-original draft preparation. XF and LY: software. XF, LY, DW, and CZ: validation. XF, LY, DW, YC, YW, ZW, RL, JR, WT, and $\mathrm{CZ}$ : investigation and resources. WT and CZ: data curation and supervision. CZ: writing-review \& editing and Project Administration. All authors contributed to the article and approved the submitted version. 


\section{FUNDING}

This work was supported by the National Key Research and Development Program of China (2018YFC1314302), the National Natural Science Foundation of China (81771450, 81471358), the Shanghai Science and Technology Commission Foundation (14411969000), the Shanghai Municipal Education Commission-Gaofeng Clinical Medicine Grant Support (20152530), the Shanghai Municipal Commission of Health and Family Planning Foundation (201540029) and the Shanghai

\section{REFERENCES}

1. Owen MJ, Sawa A, Mortensen PB. Schizophrenia. Lancet. (2016) 388:86-97. doi: 10.1016/S0140-6736(15)01121-6

2. Zhang C, Fang X, Yao P, Mao Y, Cai J, Zhang Y, et al. Metabolic adverse effects of olanzapine on cognitive dysfunction: a possible relationship between BDNF and TNF-alpha. Psychoneuroendocrinology. (2017) 81:138-43. doi: 10.1016/j.psyneuen.2017.04.014

3. Worrel JA, Marken PA, Beckman SE, Ruehter VL. Atypical antipsychotic agents: a critical review. Am J Health Syst Pharm. (2000) 57:238-55. doi: 10.1093/ajhp/57.3.238

4. Vancampfort D, Stubbs B, Mitchell AJ, De Hert M, Wampers M, Ward $\mathrm{PB}$, et al. Risk of metabolic syndrome and its components in people with schizophrenia and related psychotic disorders, bipolar disorder and major depressive disorder: a systematic review and meta-analysis. World Psychiatry. (2015) 14:339-47. doi: 10.1002/wps.20252

5. McEvoy JP, Meyer JM, Goff DC, Nasrallah HA, Davis SM, Sullivan $\mathrm{L}$, et al. Prevalence of the metabolic syndrome in patients with schizophrenia: baseline results from the clinical antipsychotic trials of intervention effectiveness (CATIE) schizophrenia trial and comparison with national estimates from NHANES III. Schizophr Res. (2005) 80:19-32. doi: 10.1016/j.schres.2005.07.014

6. Grover S, Padmavati R, Sahoo S, Gopal S, Nehra R, Ganesh A, et al. Relationship of metabolic syndrome and neurocognitive deficits in patients with schizophrenia. Psychiatry Res. (2019) 278:56-64. doi: 10.1016/j.psychres.2019.05.023

7. Olfson M, Gerhard T, Huang C, Crystal S, Stroup TS. Premature mortality among adults with schizophrenia in the United States. JAMA Psychiatry. (2015) 72:1172-81. doi: 10.1001/jamapsychiatry.2015.1737

8. Jeon SW, Kim YK. Unresolved issues for utilization of atypical antipsychotics in schizophrenia: antipsychotic polypharmacy and metabolic syndrome. Int J Mol Sci. (2017) 18:2174. doi: 10.3390/ijms18102174

9. Deng C, Weston-Green K, Huang XF. The role of histaminergic H1 and $\mathrm{H} 3$ receptors in food intake: a mechanism for atypical antipsychotic-induced weight gain? Prog Neuropsychopharmacol Biol Psychiatry. (2010) 34:1-4. doi: 10.1016/j.pnpbp.2009.11.009

10. Penninx B, Lange SMM. Metabolic syndrome in psychiatric patients: overview, mechanisms, and implications. Dialogues Clin Neurosci. (2018) 20:63-73. doi: 10.31887/DCNS.2018.20.1/bpenninx

11. Quiñones M, Al-Massadi O, Fernø J, Nogueiras R. Cross-talk between SIRT1 and endocrine factors: effects on energy homeostasis. Mol Cell Endocrinol. (2014) 397:42-50. doi: 10.1016/j.mce.2014.08.002

12. Zhou S, Tang X, Chen HZ. Sirtuins and insulin resistance. Front Endocrinol. (2018) 9:748. doi: 10.3389/fendo.2018.00748

13. Arab Sadeghabadi Z, Nourbakhsh M, Pasalar P, Emamgholipour S, Golestani A, Larijani B, et al. Reduced gene expression of sirtuins and active AMPK levels in children and adolescents with obesity and insulin resistance. Obes Res Clin Pract. (2018) 12:167-73. doi: 10.1016/j.orcp.2017.10.004

14. Mariani S, Fiore D, Basciani S, Persichetti A, Contini S, Lubrano C, et al. Plasma levels of SIRT1 associate with non-alcoholic fatty liver disease in obese patients. Endocrine. (2015) 49:711-6. doi: 10.1007/s12020-014-0465-x

15. Ren H, Shao Y, Ma X, Yang M, Liu Y, Wang Q. Expression levels of serum vasohibin-1 and other biomarkers in type 2 diabetes mellitus patients with
Municipal Commission of Health and Family Planning, Key Developing Disciplines (2015ZB0405), the Science and Technology Development Program of Nanjing Medical University (NMUB2019107).

\section{ACKNOWLEDGMENTS}

We are deeply grateful to all the patients and healthy controls participating in this study as well as to the psychiatrists for their help in the recruitment and diagnosis of schizophrenia patients.

different urinary albumin to creatinine ratios. J Diabetes Complicat. (2019) 33:477-84. doi: 10.1016/j.jdiacomp.2019.04.008

16. Chalkiadaki A, Guarente L. High-fat diet triggers inflammation-induced cleavage of SIRT1 in adipose tissue to promote metabolic dysfunction. Cell Metab. (2012) 16:180-8. doi: 10.1016/j.cmet.2012.07.003

17. Cao MM, Lu X, Liu GD, Su Y, Li YB, Zhou J. Resveratrol attenuates type 2 diabetes mellitus by mediating mitochondrial biogenesis and lipid metabolism via sirtuin type 1. Exp Ther Med. (2018) 15:576-84. doi: 10.3892/etm.2017.5400

18. Batista-Jorge GC, Barcala-Jorge AS, Silveira MF, Lelis DF, Andrade JMO, de Paula AMB, et al. Oral resveratrol supplementation improves metabolic syndrome features in obese patients submitted to a lifestyle-changing program. Life Sci. (2020) 256:117962. doi: 10.1016/j.lfs.2020.117962

19. Zhu Y, Huang JJ, Zhang XX, Yan Y, Yin XW, Ping G, et al. Qing Gan Zi Shen Tang alleviates adipose tissue dysfunction with up-regulation of SIRT1 in spontaneously hypertensive rat. Biomed Pharmacother. (2018) 105:246-55. doi: 10.1016/j.biopha.2018.05.022

20. Wisse BE. The inflammatory syndrome: the role of adipose tissue cytokines in metabolic disorders linked to obesity. J Am Soc Nephrol. (2004) 15:2792-800. doi: 10.1097/01.ASN.0000141966.69934.21

21. Crowe J, Lumb FE, Harnett MM, Harnett W. Parasite excretory-secretory products and their effects on metabolic syndrome. Parasite Immunol. (2017) 39:e12410. doi: 10.1111/pim.12410

22. Kluge M, Schuld A, Schacht A, Himmerich H, Dalal MA, Wehmeier PM, et al. Effects of clozapine and olanzapine on cytokine systems are closely linked to weight gain and drug-induced fever. Psychoneuroendocrinology. (2009) 34:118-28. doi: 10.1016/j.psyneuen.2008.08.016

23. Beumer W, Drexhage RC, De Wit H, Versnel MA, Drexhage HA, Cohen D. Increased level of serum cytokines, chemokines and adipokines in patients with schizophrenia is associated with disease and metabolic syndrome. Psychoneuroendocrinology. (2012) 37:1901-11. doi: 10.1016/j.psyneuen.2012.04.001

24. Sainz J, Prieto C, Crespo-Facorro B. Sex differences in gene expression related to antipsychotic induced weight gain. PLoS ONE. (2019) 14:e0215477. doi: 10.1371/journal.pone.0215477

25. Fang X, Wang Y, Chen Y, Ren J, Zhang C. Association between IL-6 and metabolic syndrome in schizophrenia patients treated with secondgeneration antipsychotics. Neuropsychiatr Dis Treat. (2019) 15:2161-70. doi: 10.2147/NDT.S202159

26. Klemettilä JP, Kampman $O$, Seppälä $N$, Viikki $M$, Hämäläinen $M$, Moilanen E, et al. Cytokine and adipokine alterations in patients with schizophrenia treated with clozapine. Psychiatry Res. (2014) 218:277-83. doi: 10.1016/j.psychres.2014.04.049

27. Paredes RM, Quinones M, Marballi K, Gao X, Valdez C, Ahuja SS, et al. Metabolomic profiling of schizophrenia patients at risk for metabolic syndrome. Int J Neuropsychopharmacol. (2014) 17:1139-48. doi: 10.1017/S1461145714000157

28. Klemettilä JP, Kampman O, Seppälä N, Viikki M, Hämäläinen M, Moilanen E, et al. Association study of the HTR2C, leptin and adiponectin genes and serum marker analyses in clozapine treated long-term patients with schizophrenia. Eur Psychiatry. (2015) 30:296-302. doi: 10.1016/j.eurpsy.2014.08.006

29. Xu F, Fan W, Wang W, Tang W, Yang F, Zhang Y, et al. Effects of omega-3 fatty acids on metabolic syndrome in patients with schizophrenia: a 12-week 
randomized placebo-controlled trial. Psychopharmacology. (2019) 236:12739. doi: $10.1007 / \mathrm{s} 00213-018-5136-9$

30. Dhaliwal N, Dhaliwal J, Singh DP, Kondepudi KK, Bishnoi M, Chopra K. The probiotic mixture VSL\#3 reverses olanzapine-induced metabolic dysfunction in mice. Methods Mol Biol. (2019) 2011:531-44. doi: 10.1007/978-1-4939-9554-7_31

31. Martin AN, Alexander-Miller M, Yoza BK, Vachharajani V, McCall CE. Sirtuin1 targeting reverses innate and adaptive immune tolerance in septic mice. J Immunol Res. (2018) 2018:2402593. doi: 10.1155/2018/2 402593

32. Yu Q, Dong L, Li Y, Liu G. SIRT1 and HIFlalpha signaling in metabolism and immune responses. Cancer Lett. (2018) 418:20-6. doi: 10.1016/j.canlet.2017.12.035

33. Sadeghabadi ZA, Ziamajidi N, Abbasalipourkabir R, Mohseni R, Borzouei S. Palmitate-induced IL6 expression ameliorated by chicoric acid through AMPK and SIRT1-mediated pathway in the PBMCs of newly diagnosed type 2 diabetes patients and healthy subjects. Cytokine. (2019) 116:106-14. doi: 10.1016/j.cyto.2018.12.012

34. Asgary S, Karimi R, Momtaz S, Naseri R, Farzaei MH. Effect of resveratrol on metabolic syndrome components: a systematic review and meta-analysis. Rev Endocr Metab Disord. (2019) 20:173-86. doi: 10.1007/s11154-019-09494-z

35. Cheng Y, Wu W, Wang J, Feng W, Wu X, Li C. Reliability and validity of the repeatable battery for the assessment of neuropsychological status in community-dwelling elderly. Arch Med Sci. (2011) 7:850-7. doi: 10.5114 /aoms.2011.25561

36. Fang X, Chen Y, Wang Y, Zhang C. Identification of risk factors for suicidal ideation in patients with schizophrenia. Psychiatry Res. (2019) 271:195-9. doi: 10.1016/j.psychres.2018.11.051

37. Leucht S, Samara M, Heres S, Davis JM. Dose equivalents for antipsychotic drugs: the DDD method. Schizophr Bull. (2016) 42 (Suppl. 1):S90-4. doi: $10.1093 / \mathrm{schbul} / \mathrm{sbv} 167$

38. Postolache TT, Del Bosque-Plata L, Jabbour S, Vergare M, Wu R, Gragnoli C. Co-shared genetics and possible risk gene pathway partially explain the comorbidity of schizophrenia, major depressive disorder, type 2 diabetes, and metabolic syndrome. Am J Med Genet B Neuropsychiatr Genet. (2019) 180:186-203. doi: 10.1002/ajmg.b.32712

39. Casarotto AAF, Galera BB, Sumiyoshi LM, Floor TM. Polymorphism rs7895833 in the SIRT1 gene and its association with dyslipidaemia in the elderly. Rev Esp Geriatr Gerontol. (2019) 54:214-9. doi: 10.1016/j.regg.2019.01.008

40. Li F, Li H, Jin X, Zhang Y, Kang X, Zhang Z, et al. Adipose-specific knockdown of Sirtl results in obesity and insulin resistance by promoting exosomes release. Cell Cycle. (2019) 18:2067-82. doi: 10.1080/15384101.2019.1638694

41. Zhong XL, Miao HJ, Fang ZM, Kuken B, Song HY, Zhong H, et al. The effect of SIRT1 gene polymorphisms on ambulatory blood pressure of hypertensive patients in the kazakh population. Genet Test Mol Biomarkers. (2015) 19:5615. doi: $10.1089 / \mathrm{gtmb} .2015 .0111$

42. Wang Y, Huang Y, Peng M, Cong Z, Li X, Lin A, et al. Association between silent information regulator 1 (SIRT1) gene polymorphisms and schizophrenia in a Chinese han population. Psychiatry Res. (2015) 225:744-5. doi: 10.1016/j.psychres.2014.11.027

43. Wawryka J, Barg E. [Impact of SIRT1 gene expression on the development and treatment of the metabolic syndrome in oncological patients]. Pediatr Endocrinol Diabetes Metab. (2016) 22:60-5. doi: 10.18544/PEDM-22.02.0052

44. Gao D, Zuo Z, Tian J, Ali Q, Lin Y, Lei H, et al. Activation of SIRT1 attenuates klotho deficiency-induced arterial stiffness and hypertension by enhancing AMP-activated protein kinase activity. Hypertension. (2016) 68:1191-9. doi: 10.1161/HYPERTENSIONAHA.116.07709

45. Tain YL, Lin YJ, Sheen JM, Lin IC, Yu HR, Huang LT, et al. Resveratrol prevents the combined maternal plus postweaning high-fat-dietsinduced hypertension in male offspring. J Nutr Biochem. (2017) 48:120-7. doi: 10.1016/j.jnutbio.2017.06.007
46. Rogacka D, Piwkowska A, Audzeyenka I, Angielski S, Jankowski M. SIRT1AMPK crosstalk is involved in high glucose-dependent impairment of insulin responsiveness in primary rat podocytes. Exp Cell Res. (2016) 349:328-38. doi: 10.1016/j.yexcr.2016.11.005

47. Aditya R, Kiran AR, Varma DS, Vemuri R, Gundamaraju R. A review on SIRtuins in diabetes. Curr Pharm Des. (2017) 23:2299-307. doi: 10.2174/1381612823666170125153334

48. Gok O, Karaali Z, Ergen A, Ekmekci SS, Abaci N. Serum sirtuin 1 protein as a potential biomarker for type 2 diabetes: increased expression of sirtuin 1 and the correlation with microRNAs. J Res Med Sci. (2019) 24:56. doi: 10.4103/jrms.JRMS_921_18

49. Rahimi M, Ghanbari S, Khazaei M, Niroman E. Comparison of sirtuin 1 level and related blood factors in diabetic and healthy subjects. Pediatr Endocrinol Diabetes Metab. (2020) 26:17-21. doi: 10.5114/pedm.2020.94392

50. Victoriano M, de Beaurepaire R, Naour N, Guerre-Millo M, QuignardBoulangé A, Huneau JF, et al. Olanzapine-induced accumulation of adipose tissue is associated with an inflammatory state. Brain Res. (2010) 1350:167-75. doi: 10.1016/j.brainres.2010.05.060

51. Zhang Q, He M, Deng C, Wang H, Huang XF. Effects of olanzapine on the elevation of macrophage infiltration and pro-inflammatory cytokine expression in female rats. J Psychopharmacol. (2014) 28:1161-9. doi: $10.1177 / 0269881114555250$

52. Zhang Y, Fang X, Fan W, Tang W, Cai J, Song L, et al. Brainderived neurotrophic factor as a biomarker for cognitive recovery in acute schizophrenia: 12-week results from a prospective longitudinal study. Psychopharmacology. (2018) 235:1191-8. doi: 10.1007/s00213-0184835-6

53. Sirota P, Hadi E, Djaldetti M, Bessler H. Difference in inflammatory cytokine production by mononuclear cells from obese and non-obese schizophrenic patients. Acta Psychiatr Scand. (2015) 132:301-5. doi: 10.1111/acps. 12396

54. Zhang C, Zhang Y, Cai J, Chen M, Song L. Complement 3 and metabolic syndrome induced by clozapine: a cross-sectional study and retrospective cohort analysis. Pharmacogenomics J. (2017) 17:92-7. doi: 10.1038/tpj.2015.68

55. Hui X, Zhang M, Gu P, Li K, Gao Y, Wu D, et al. Adipocyte SIRT1 controls systemic insulin sensitivity by modulating macrophages in adipose tissue. EMBO Rep. (2017) 18:645-57. doi: 10.15252/embr.201643184

56. Kim SY, Lee MS, Chang E, Jung S, Ko H, Lee E, et al. Tartary buckwheat extract attenuated the obesity-induced inflammation and increased muscle PGC1a/SIRT1 expression in high fat diet-induced obese rats. Nutrients. (2019) 11:654. doi: 10.3390/nu11030654

57. Inoue $T$, Tanaka M, Masuda $S$, Ohue-Kitano R, Yamakage H, Muranaka $\mathrm{K}$, et al. Omega-3 polyunsaturated fatty acids suppress the inflammatory responses of lipopolysaccharide-stimulated mouse microglia by activating SIRT1 pathways. Biochim Biophys Acta Mol Cell Biol Lipids. (2017) 1862:55260. doi: 10.1016/j.bbalip.2017.02.010

58. Peng J, Zhou Y, Hong Z, Wu Y, Cai A, Xia M, et al. Maternal eicosapentaenoic acid feeding promotes placental angiogenesis through a sirtuin-1 independent inflammatory pathway. Biochim Biophys Acta Mol Cell Biol Lipids. (2019) 1864:147-57. doi: 10.1016/j.bbalip.2018.11.003

Conflict of Interest: The authors declare that the research was conducted in the absence of any commercial or financial relationships that could be construed as a potential conflict of interest.

Copyright $\odot 2020$ Fang, Yu, Wang, Chen, Wang, Wu, Liu, Ren, Tang and Zhang. This is an open-access article distributed under the terms of the Creative Commons Attribution License (CC BY). The use, distribution or reproduction in other forums is permitted, provided the original author(s) and the copyright owner(s) are credited and that the original publication in this journal is cited, in accordance with accepted academic practice. No use, distribution or reproduction is permitted which does not comply with these terms. 